\title{
Faults detection and diagnoses of permanent magnet synchronous motor based on neuro-fuzzy network
}

\author{
Hashmia Sh. Dakheel, Zainab B. Abdulla, Helen Jassim Jawad \\ Electromechanical Department, University of Technology, Iraq
}

\begin{tabular}{|c|c|}
\hline Article Info & ABSTRACT \\
\hline Article history: & Faults in electrical machine are very important in order to improve the \\
\hline Received Dec 19, 2018 & $\begin{array}{l}\text { machine expensive maintenance, efficiency, life time, and reliability at real } \\
\text { time, therefore this study deals with Simulink model response for healthy and }\end{array}$ \\
\hline Revised Feb 14, 2019 & neuro-fuzzy network (ANFIS), this intelligent technique consist of two parts, \\
\hline Accepted Mar 1, 2019 & $\begin{array}{l}\text { the first part include electrical and demagnetization faults while another part } \\
\text { deals with mechanical faults. Simulation results obtain record activation, }\end{array}$ \\
\hline Keywords: & \\
\hline
\end{tabular}

ANFIS

Fault detect and diagnoses

Motor system

Neuro-fuzzy

Permanent magnet

Copyright $\odot 2019$ Institute of Advanced Engineering and Science. All rights reserved.

Corresponding Author:

Hashmia Sh. Dakheel,

Electromechanical Department,

University of Technology,

Al-Sina'a Street, 35010, Baghdad, Iraq

Email: hashimia.shr@yahoo.com

\section{INTRODUCTION}

Permanent magnet synchronous motors (PMSMs) have high specifications in applications required high efficiency, low inertia, high power density, high second ratio and high torque to current ratio. [1]; therefore PMSM are widely used in industrial applications such as machine tool, winders, robots and the systems required torque and speed control in high accuracy and capability to work at multi speeds and occasional inertia [2-3].Periodic preservation will increase motor life styles in machines along with PMSM. Latterly, programs engineers and scientists have targeted on predictive protection in preference to periodic preservation [4]. The failure of a force in distinct packages has a series effect at the operation of a system, the failure results on occasion in misplaced manufacturing; while in others it may have an effect on safety of human. [5].

In engineering structures to avoid severe consequence, in complicated systems any fault possesses the potential to impact the entire systems behavior even as in a production procedure simple fault in off specification products, close down of production lines, better operations cost, and environmental harm, and so on[6].At recent days, the demand increasing in industrial applications by operators and users to detect and diagnoses faults that occur in electric machine during operation to achieve high reliability and safety [7-8]. The neuro-fuzzy technique can be obtained by combination between artificial neural network (ANN) and fuzzy controller, these techniques have properties of fuzzy rules and sets are adjusted by used (ANN) [9]. This paper studies speed characteristic and electromechanical torque at electrical, mechanical and demagnetization faults conditions in PMSM by implementation an intelligent approach neuro-fuzzy network .Adaptive neuro-fuzzy inference system(ANFIS) - based agents are trained using simulation results to automate the process. Which is a developed tool use to detect and diagnosis electrical, mechanical and demagnetization faults in PMSM in order to avoid these faults and enhance efficiency and life time. 


\section{MATHEMATICAL MODEL OF PMSM:-} PMSM are:

The direct $(d)$ and quadrature $(q)$ variables of voltage equations formula in a rotor reference frame of

$V d=R_{s} i_{d}+\frac{\mathrm{d}\left(\phi_{d}\right)}{\mathrm{d} t}-\omega \phi_{q}$

$V q=R_{s} i_{d}+\frac{\mathrm{d}\left(\phi_{d}\right)}{\mathrm{d} t}+\omega \phi_{d}+\omega \phi_{f}$

That components of the stator flux linkage are:-

$\phi_{d}=L_{d} i_{d}+\phi_{f}$

$\phi_{q}=L_{q} i_{q}$

Where $R_{S}$ is the resistance of stator winding, $V q$ is q- axis voltage and $V d$ is d -axis voltage, $\phi_{d}$ is d-axis flux and $\phi_{q}$ is q-axis flux, $\phi_{f}$ is the rotor flux linkage generated by the permanent magnet, $i_{d}$ is d-axis current and $i_{q}$ is q- axis current, $\omega$ is the angular speed in the rotor, $L_{q}$ and $L_{d}$ are q-d axis inductances. Mechanical torque in motor at load is given by:

$T_{e}=\left(\frac{J}{P}\right) d \omega+\left(\frac{D}{P}\right) \omega+T_{L}$

With

$T_{e}=3 / 2 P\left(\phi_{d} i_{d}+\left(L_{d}-L_{q}\right) i_{d} i_{q}\right)$

Where $J$ is the moment of the inertia in rotor, $P$ is the number of pole pairs, $T_{L}$ is the load torque, and $D$ is the damping of torque coefficient [10].

\section{NEURAL-FUZZY NETWORKS}

\subsection{Fuzzy logic controller (FLC)}

This type is a class of artificial intelligence with a latest history and alertness. Fuzzy logic presents a simple manner to reach at a particular end based totally upon vague, ambiguous, obscure, noisy, or lacking input data. Its approach to control problems mimics how a person would make selections, a good deal faster [11].

\subsection{Artificial neural networks (ANN)}

It is kind of synthetic intelligence method in the beginning designed to mimic the functionality of the human mind. A neural network is composed of many easy and particularly interconnected processors known as neuroses. These are analogous to the biological neurons inside the human mind. The artificial neuroses are linked by hyperlinks that bring signals among each other, just like biological neurons. The neuroses receive input stimuli which can be translated into an output stimulus [12]. ANN has essentially defined as the knowledge required by the network from its environment through a learning process and synaptic weight are used to store acquired knowledge [13].

\subsection{Neuro-fuzzy networks}

This network is a fuzzy system this is educated by a learning set of rules derived from neural community principle. In neuro-fuzzy models, connection weights, and propagation and activation functions fluctuate from commonplace neural networks. This learning procedure work on local information, and causes only local changes in the fuzzy device [14]. 


\subsection{Adaptive neuro-fuzzy inference system (ANFIS)}

It is one types of neuro-fuzzy network, it based on a fuzzy system which is trained by a learning algorithm derived from neural network theory, while the learning capability is advantage from view point of FIS , the formation of linguistic rules base will advantage from the viewpoint of ANN, there are several approaches to integrate ANN and FIS and very often the choice depends on the application [15], the implementation of fuzzy inference mechanisms on adaptive network architectures enables the optimization of system parameters to match targeted non-linear performance. ANFIS abilities to perform pattern recognition, nonlinear mapping and system identification make it an outstanding candidate to automate the machine fault diagnosis.[16]Among many FIS models the sugeuo fuzzy model is the most widely applied one for high interpret ability and computational efficiency and built-in optimal and adaptive techniques. ANFIS consists of two input namely $\mathrm{X}$ and $\mathrm{Y}$ and one output $(\mathrm{Z})$ and there are two rules:

Rule 1: if $\mathrm{x}$ is $\mathrm{A} 1$ and $\mathrm{y}$ is $\mathrm{B} 1$, then $\mathrm{z} 1=\mathrm{p} 1 \mathrm{x}+\mathrm{q} 1 \mathrm{y}+\mathrm{r} 1$

Rule 2: if $\mathrm{x}$ is $\mathrm{A} 2$ and $\mathrm{y}$ is $\mathrm{B} 2$, then $\mathrm{z} 2=\mathrm{p} 2 \mathrm{x}+\mathrm{q} 2 \mathrm{y}+\mathrm{r} 2$,

Where $\mathrm{Ai}$ and $\mathrm{Bi}$ are the fuzzy sets in the antecedent, and pi, qi and ri are the design parameters that are determined during the training process [17]. The structure of ANFIS consists of five layers:

Layer 1: consists of input variables (membership functions) and triangular or bell shaped membership functions.

Layer 2: is membership layer and it checks for the weights of each membership functions. It receives the input values from the first layer and act as membership functions to represent the fuzzy sets of the respective input variables.

Layer 3: is called as rule layer and it receives input from the previous layer. Each node (each neuron) in this layer performs the pre-condition matching of the fuzzy rules. This layer computes the activation level of each rule and the number of layers equals to the number of fuzzy rules. Each node of this layer calculates the weights which will be normalized.

Layer 4: is the defuzzification layer which provides the output values resulting from the inference of rules.

Layer 5: is called as the output layer which sums up all the inputs coming from layer 4 and transforms the fuzzy classification results into a crisp value, the structure of ANFIS can be shown in Figure 1 [18]. And the proposed ANFIS based detection and diagnosis can be shown in Figure 2 and Figure 3 shows simulink model.

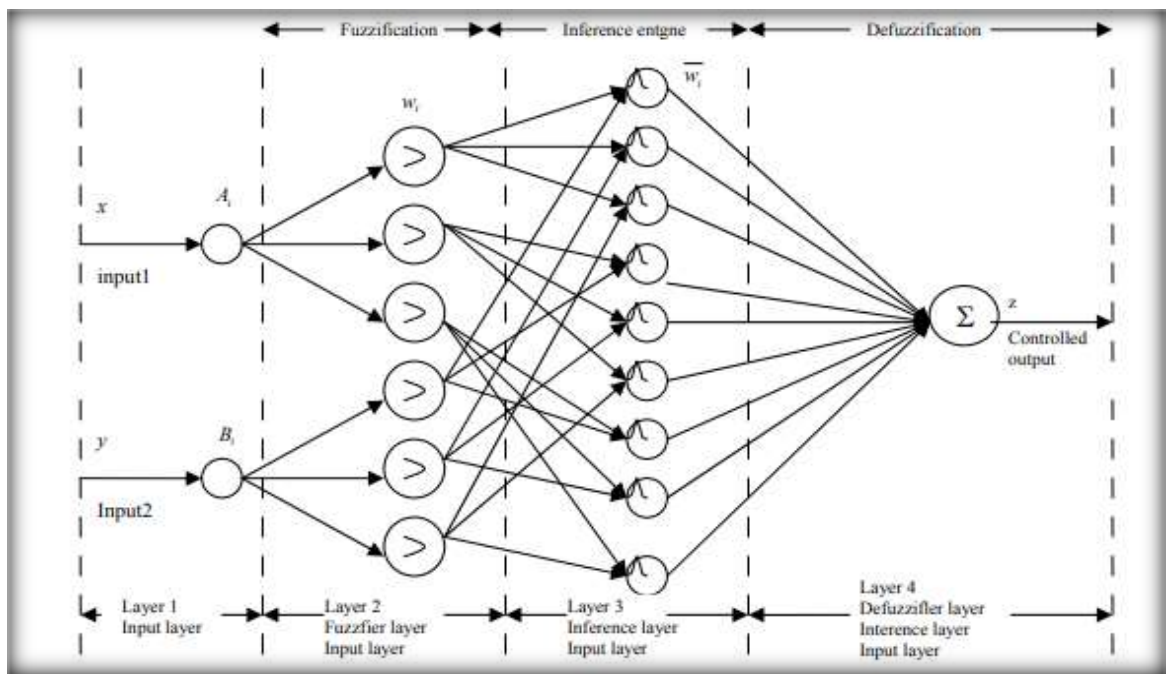

Figure 1. ANFIS structure

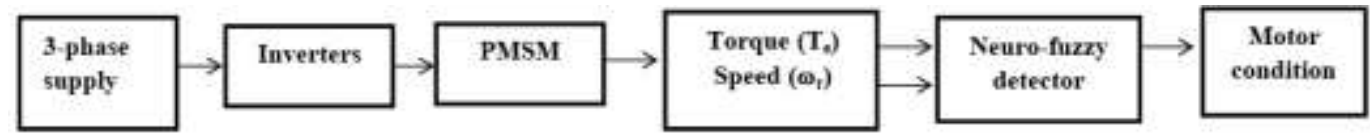

Figure 2. System of detection and diagnosis faults in PMSM conditions

Faults detection and diagnoses of permanent magnet synchronous motor based ... (Hashmia Sh. Dakheel) 


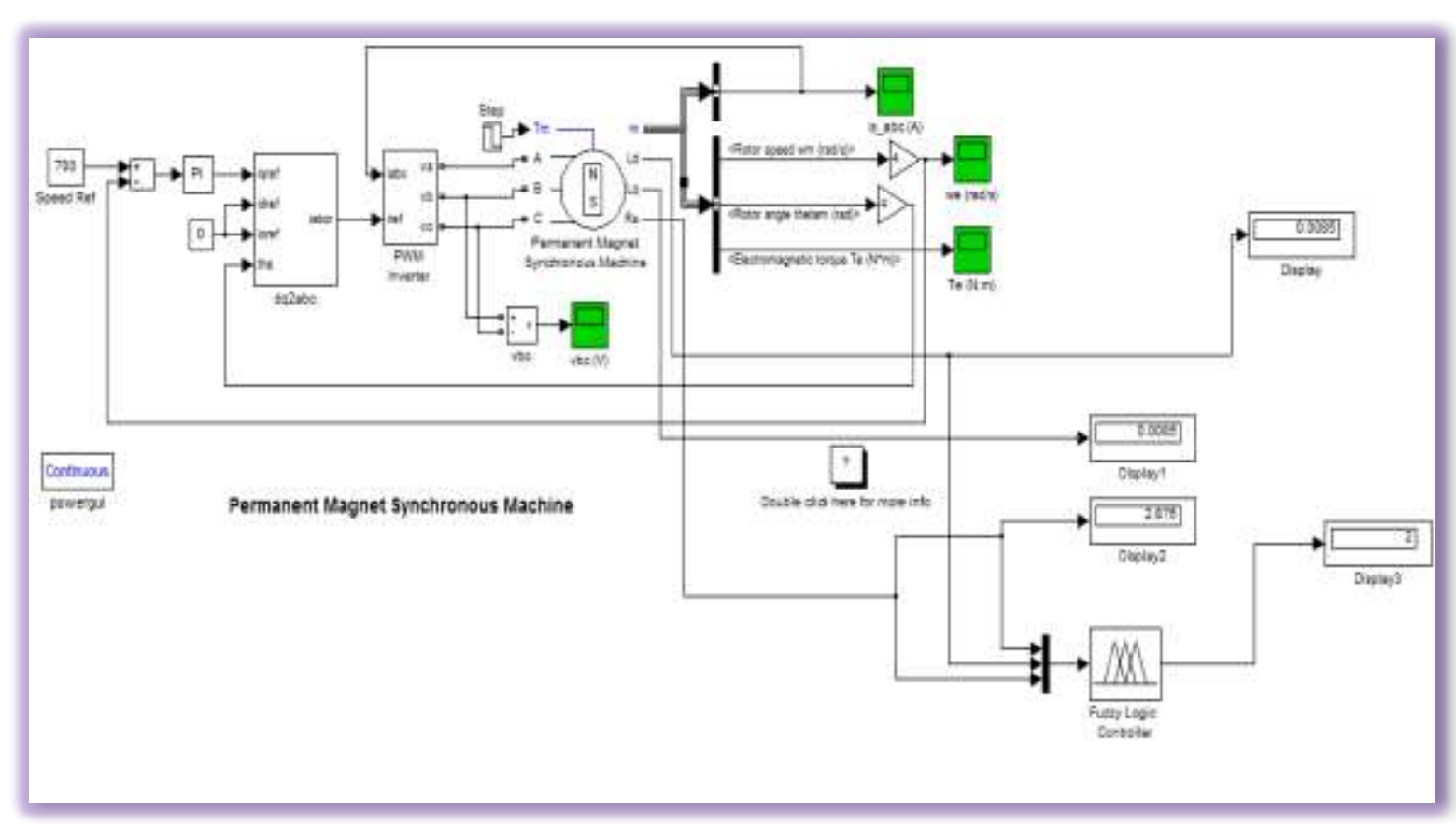

Figure 3. Simulink model for detection and diagnosis faults in PMSM

\section{FAULT IN PMSM}

Its very necessary to achieve increase performance, raise life time, and smaller costs. In general the faults occur in PMSM can be classified into the following: 1-Electrical faults, 2-mechanical faults, 3magnetic (demagnetization) faults [19], these faults can be shown in the below chart

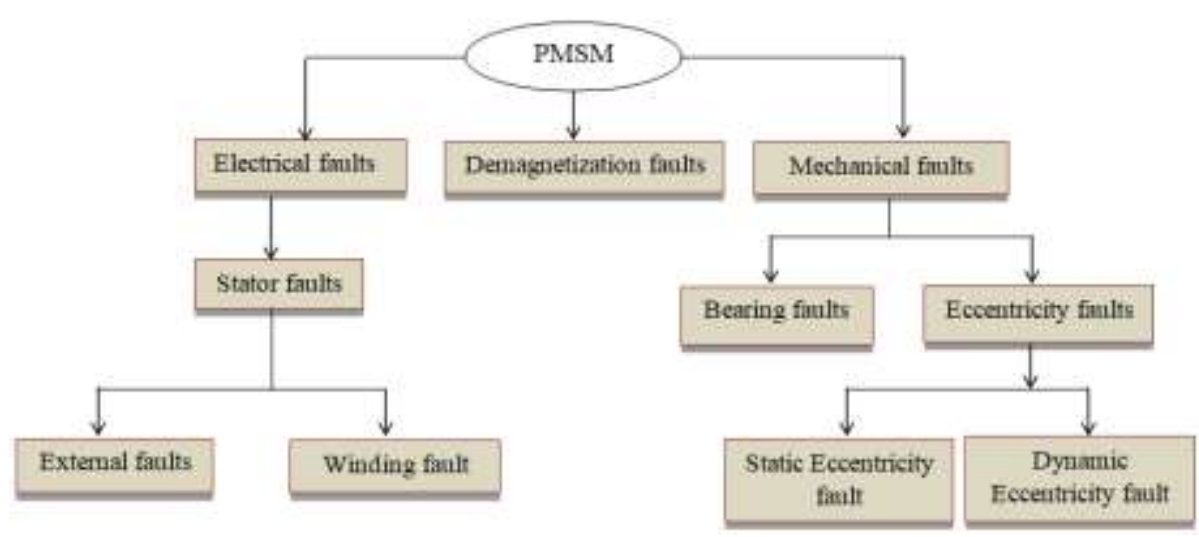

Figure 4. Classification of fault in PMSM

\subsection{Electrical faults}

Stator faults occur because insulation failure, there are different types of these faults, about 35 to 40 percentage of motors are failure related to stator winding insulation and core. This fault causes high current flow and generate excessive heat in shorted turn similar to 3-phase ac machine [14]. The abnormal variation of $\mathrm{R}_{\mathrm{s}}$ and $\mathrm{L}_{\mathrm{q}}$ causes the electrical fault that increases heating of the generating winding which leads to reduce the efficiency in motor, Figure 4-5 show the characteristics of PMSM (electromechanical torque and speed respectively)at healthy conditions, and Figure 6-7 show the characteristics under electrical fault (variation of $\mathrm{R}_{\mathrm{s}}$ and $\mathrm{L}_{\mathrm{q}}$ ). 


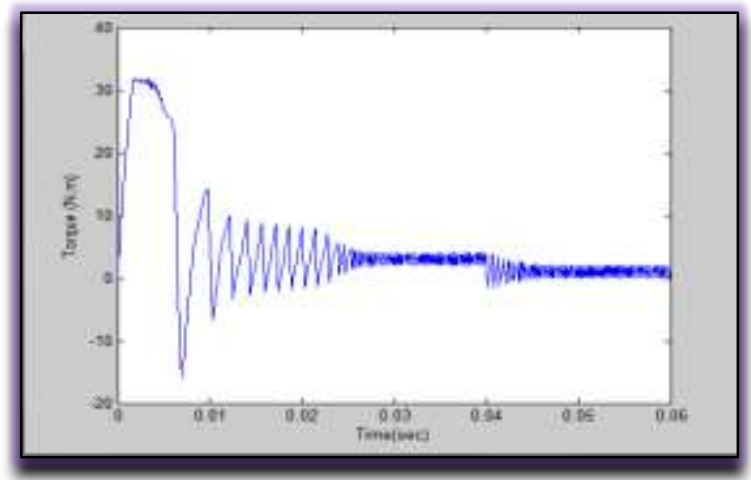

Figure 4. Electromechanical torque at healthy conditions

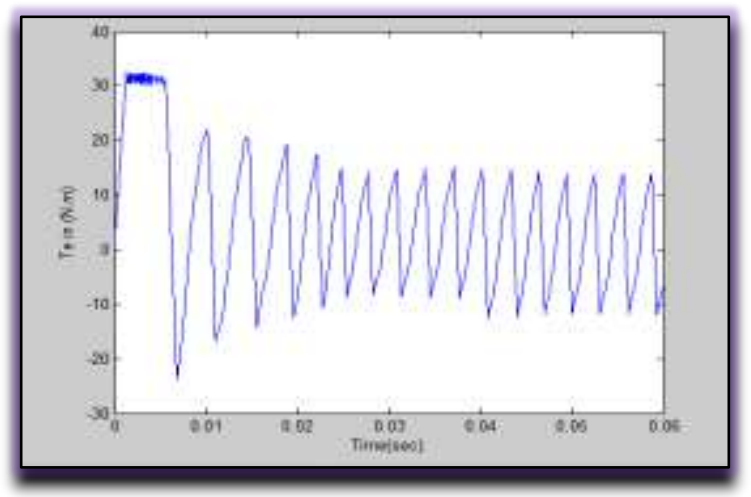

Figure 6. Electromechanical torque at electrical faults conditions

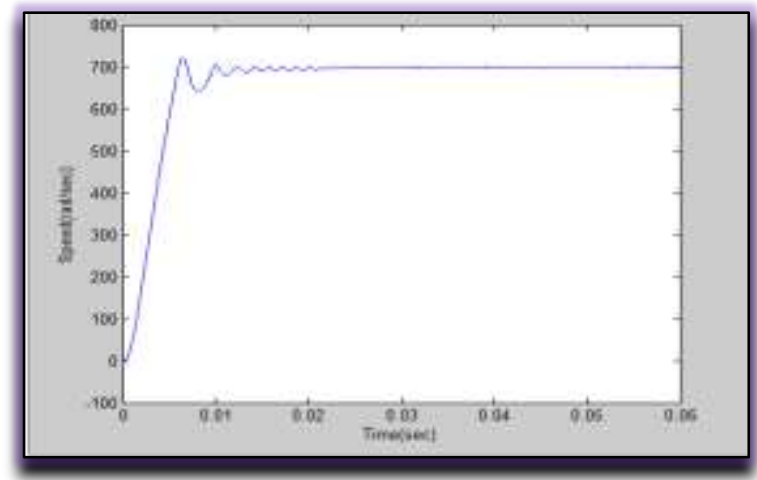

Figure 5. Speed characteristic at healthy conditions

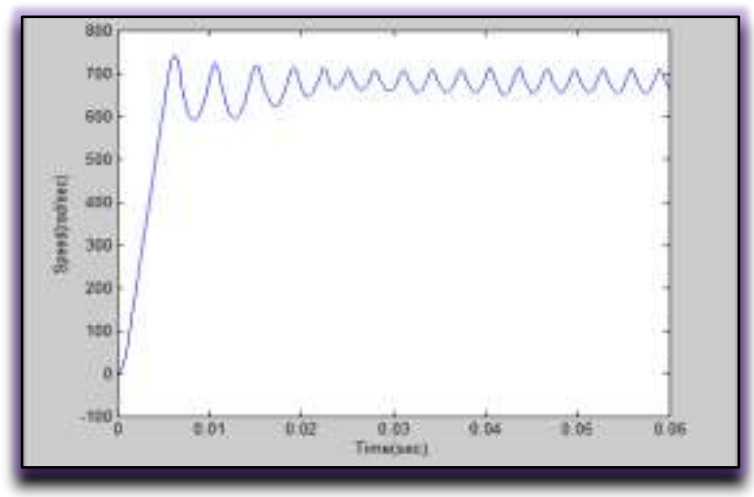

Figure 7. Speed characteristic at electrical faults conditions

\subsection{Demagnetization faults}

This is the magnetic fault, that can occur because a combination of electrical, mechanical, thermal and environmental stress on the motor leading_to other faults can occur because some defections in permanent magnet caused by mechanical or environmental stresses leading to chipped or cracked magnets producing an air gap flux disturbance similar to a local demagnetization [20-21], this type of faults usually occurs during fast change from stationary to transient states. In opposite directions of magnetic fields are caused high currents passing through stator coils and high temperature in winding faults [22]. Figure 8-9 show the characteristics of PMSM at demagnetization faults.

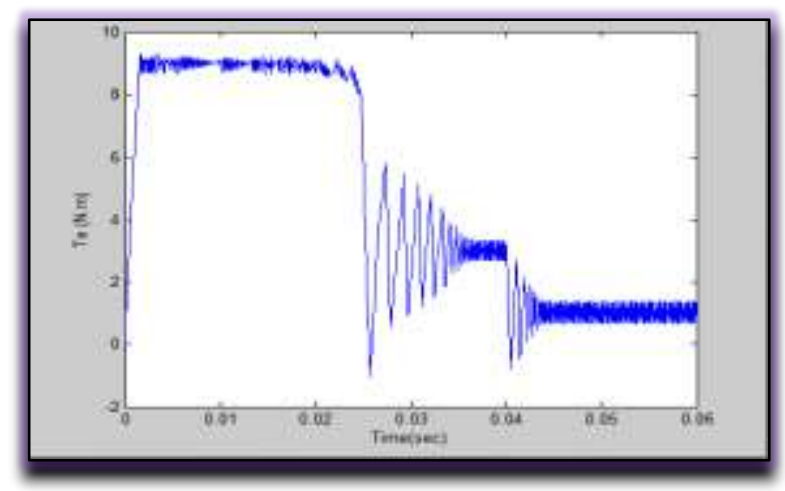

Figure 8. Electromechanical torque at demagnetization faults conditions

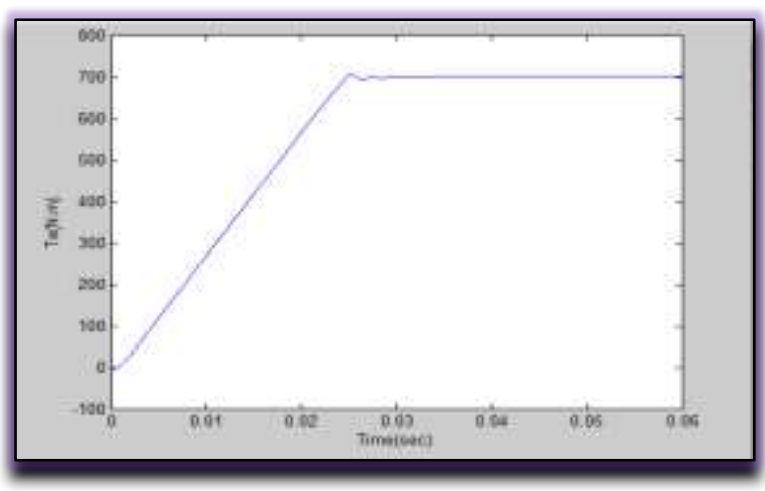

Figure 9. Speed characteristic at demagnetization faults conditions 


\subsection{Mechanical fault}

The mechanical fault in PMSM occurs due to a fault in bearing, difference fluxes in air gap, motor eccentricity, and deflection in shaft or mass unbalance [23].

\section{- Bearing fault}

The bearing fault is a mechanical fault which can arise in PMSMs. This fault is caused by production defects, the motor shaft voltages, some environmental which includes excessive temperature and humidity, factors which includes improper installation and insufficient lubrication. While the selection of switching frequency high inside the motor force gives noiseless operation of the motor, it speedy causes defects within the bearings [5].

\section{- Eccentricity fault}

This fault occurs when unequal air gap between the rotor and stator, eccentricity in air gap can be introduced during imperfections in the manufacturing stage or motor operation [20]. Incorrect positioning for eccentricity faults that caused the bending of the motor shaft. This fault can be classified into three types: static eccentricity (SE), dynamic eccentricity (DE), and mixed eccentricity which is tack place from static and dynamic simultaneously[2], static eccentricity is occur when the air gap minimum radial of the position is fixed. It can be caused by the incorrect positioning of the stator middle or the stator core ovality, at the same time as dynamic eccentricity is arise in which the location of the minimum radial air gap rotates with rotor. This fault can be caused by a dishonest shaft, worn bearings, uneven thermal expansion of the rotor, and excessive degree of static eccentricity, eccentricity produces unbalanced magnetic pull, which ends up in bearing put on, vibration, rotor deflection and acoustic noise [20]. In another mean (DE) faults occur when the center of rotor and the center of rotation are not identical while (SE) occurs when the centerline does not synchronous with the stator bore these faults can be shown in Figure 10 [24].

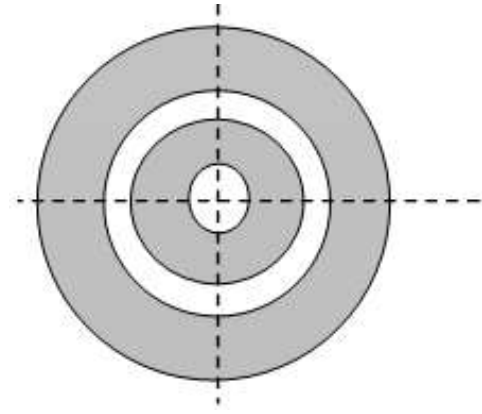

Centric rotor

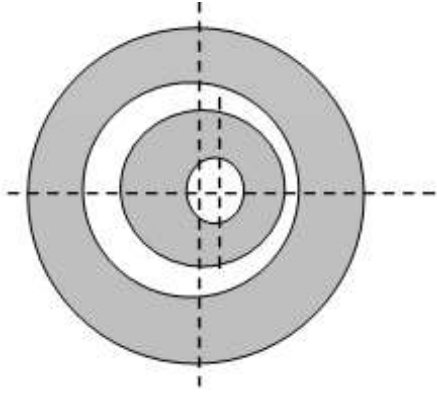

Static eccentricity (SE)

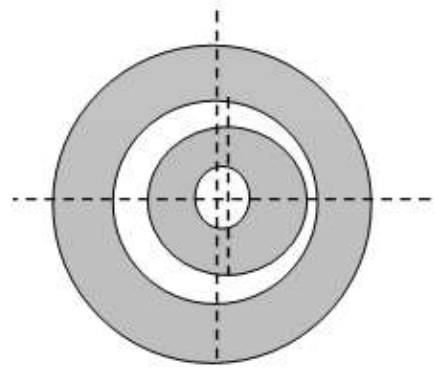

Dynamic eccentricity (DE)

Figure 10. Eccentricity faults

\section{SIMULATION RESULTS OF PMSM}

The simulation results of this study include the conditions of the PMSM, the model consists of two parts, the first part refers to electrical and demagnetization faults while another part contains mechanical fault.

\subsection{Electrical and demagnetization faults}

The training data by using neuro-fuzzy network and neural artificial network of the simulink model can be shown in Figure 11 and 12.

This intelligent network consists of three inputs $(\mathrm{Rs}-\mathrm{Lq}-\phi)$ and one output conditions of machine (healthy or faults conditions)) and 27 hidden layers of the network that represented 27 rules of fuzzy detectors. 


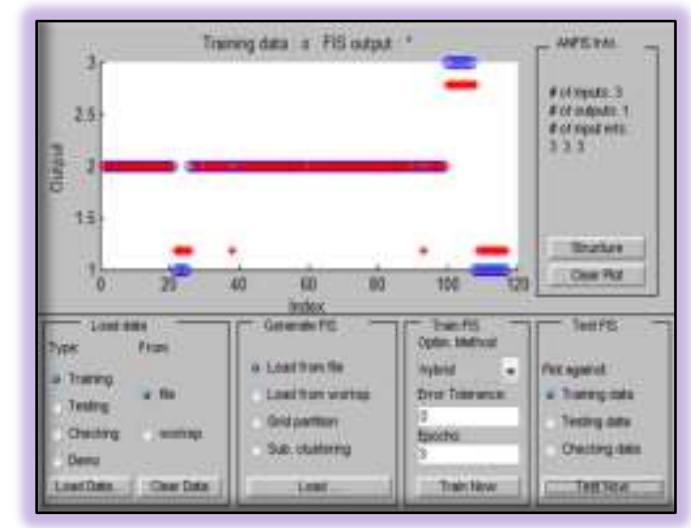

Figure 11. Training data of neural fuzzy at electrical and demagnetization faults conditions

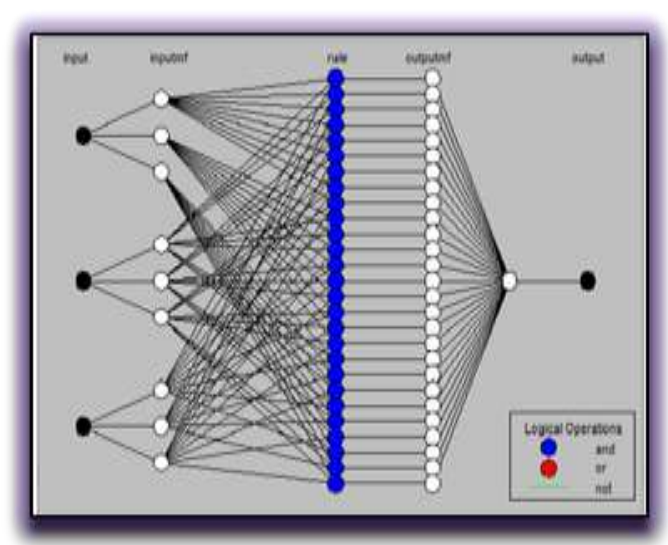

Figure 12. Neural artificial network

Figure 13 represents the simulation results of the proposed model at healthy condition when the value of parameters at rated value (input1 $\left(\mathrm{R}_{\mathrm{s}}\right)=2.85 \Omega$, input $2\left(\mathrm{~L}_{\mathrm{q}}=8.75 \mathrm{e}-3\right)$ and input 3 (flux $\left.\phi=0.175 \mathrm{wb}\right)$ then the output of the model $=1$ (normal condition), while Figure (14) represents electrical fault when $R_{s}$ varying from $\left(2.85\right.$ to $8.49 \Omega$ ) and Figure 15 at $\mathrm{L}_{\mathrm{q}}$ varying to $(16 \mathrm{e}-4)$, the output $=2$ which represents electrical fault.

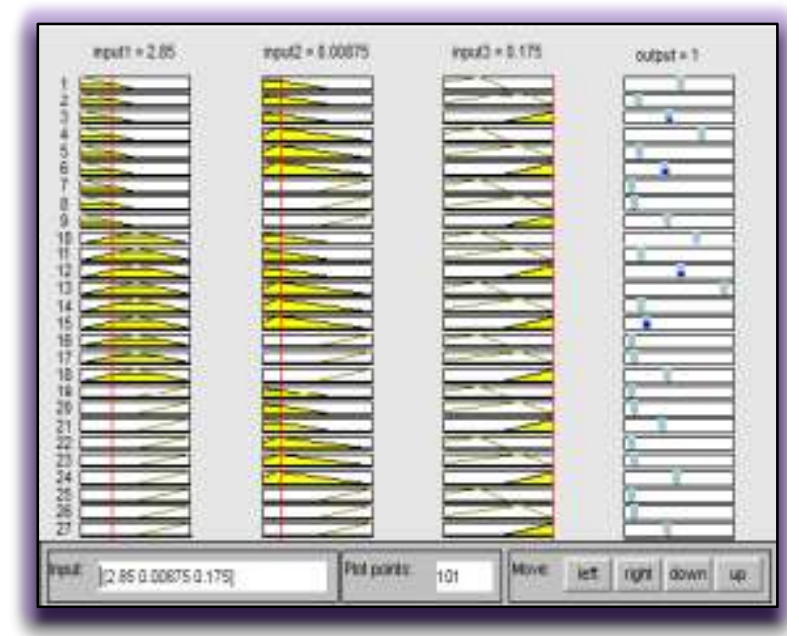

Figure 13. Results simulation at healthy condition of PMSM

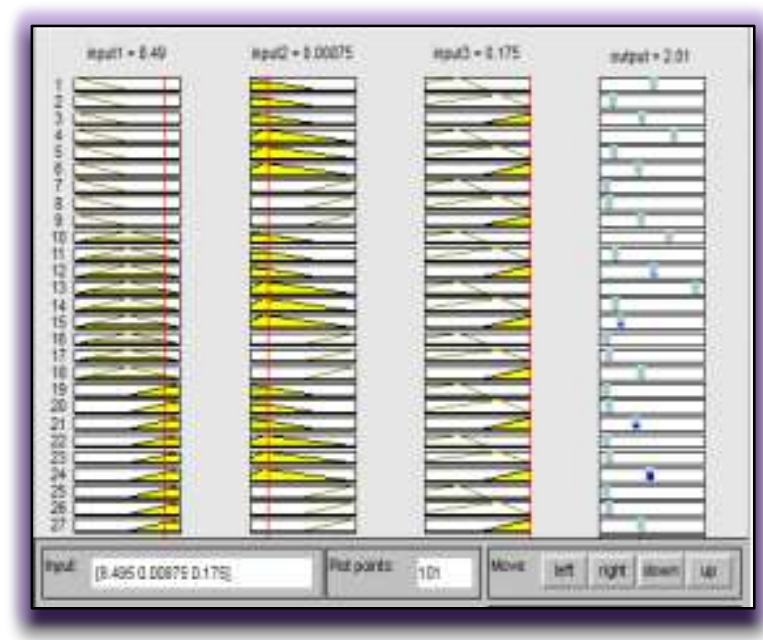

Figure 14. Results simulation at electrical fault condition (change in Rs value)

Demagnetization fault introduced by varying flux (decrease flux), Figure 16 shows the simulation results of this fault at (input $1=(\mathrm{Rs}=2.83 \Omega)$, input $2=(\mathrm{Lq}=8.75 \mathrm{e}-3)$, input $3=$ flux $=3.48 \mathrm{e}-2 \mathrm{wb})$. However, when the flux decreases than rated value $=0.175 \mathrm{wb}$ ), the output of the detector records ( 3 ) that is referred to demagnetization fault. 


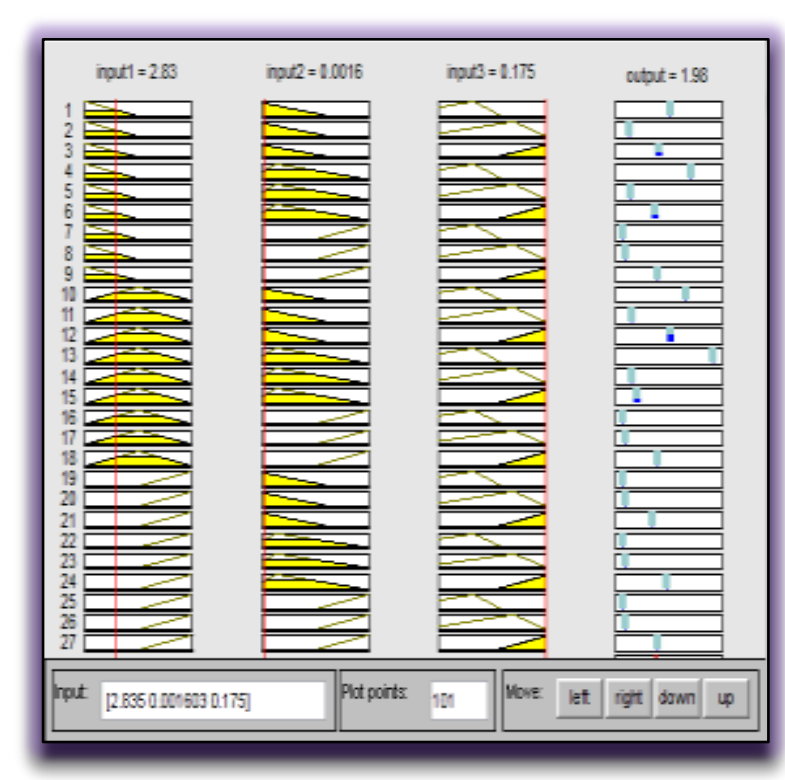

Figure 15. Simulation results at electrical fault condition (change in Lq value)

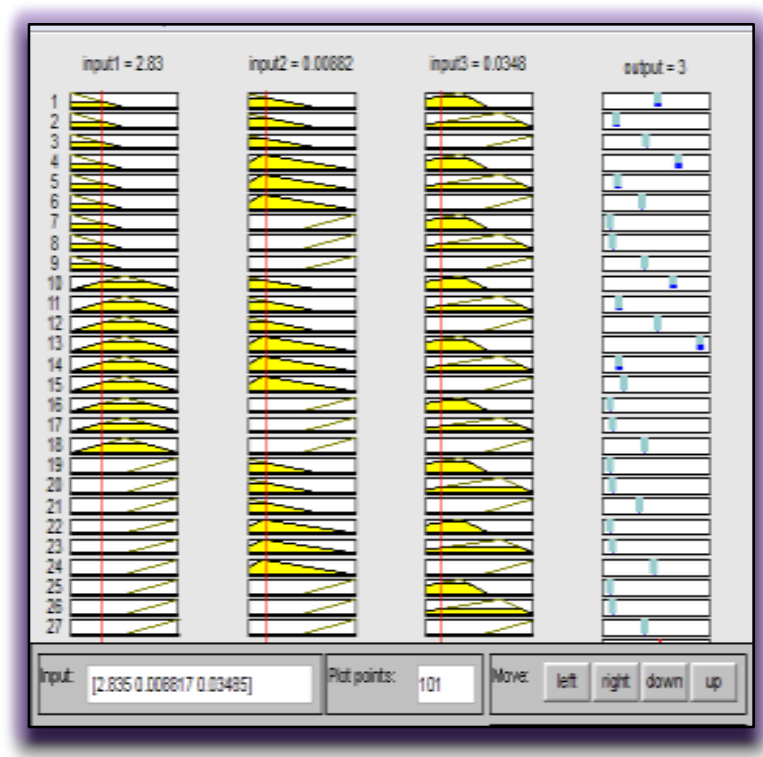

Figure 16. Results simulation at demagnetization fault condition (change in flux value)

\subsection{At mechanical fault}

The second part of the model includes mechanical fault that have two input (moment of inertia $(\mathrm{J})$ and friction factor $\left(\mathrm{B}_{\mathrm{m}}\right)$ ), (9) hidden layers (rules of fuzzy detector) and one output represents the condition of machine at healthy or mechanical fault .Figure 17 shows artificial neural network of this model.

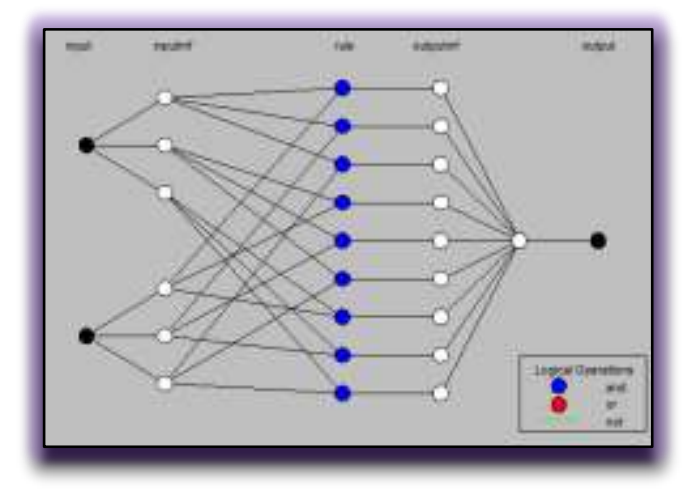

Figure 17. Neural artificial network at mechanical fault

This type of fault occurs due to the unanticipated variation of moment inertia $(J)$ and friction coefficient $\left(\mathrm{B}_{\mathrm{m}}\right)$, in case of faulty bearing the friction caused by the bearing will be high, Figure 18)-19 represent electromechanical torque and speed at mechanical fault condition. Figure 20 shows the simulation results of detector at mechanical fault when (input $1=$ moment inertia) change from rated value $(0.8 \mathrm{e}-3)$ to $(0.0537)$ and (input $2=$ friction coefficient $=0)$, the output $=4$. Also Figure 21 illustrated mechanical fault (output $=4$ ) when change $\mathrm{B}_{\mathrm{m}}$ from 0 to $(0.166)$. 


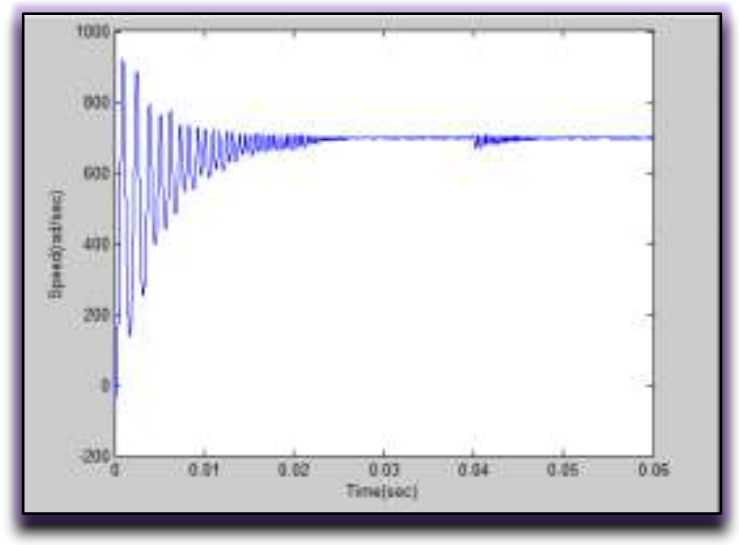

Figure 18. Speed characteristic at mechanical faults conditions

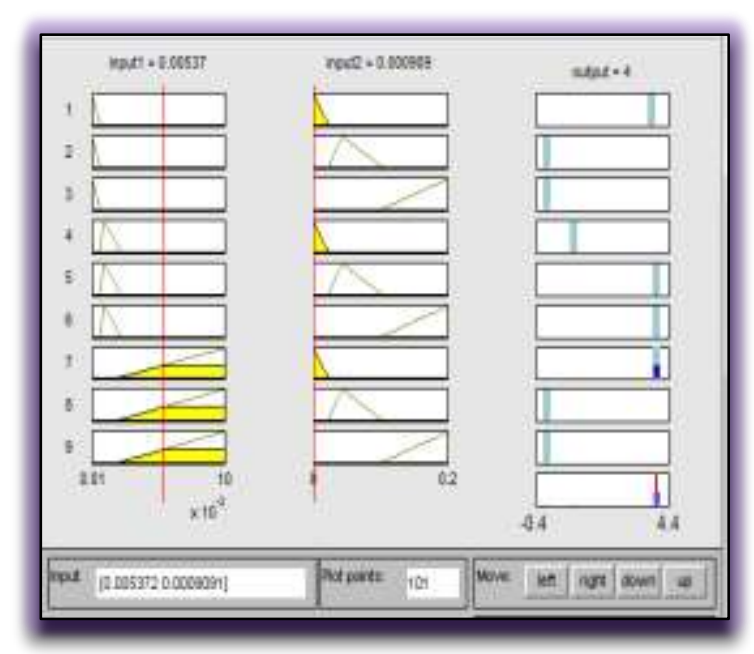

Figure 20. The results at mechanical fault (at changing $\mathrm{J}$ )

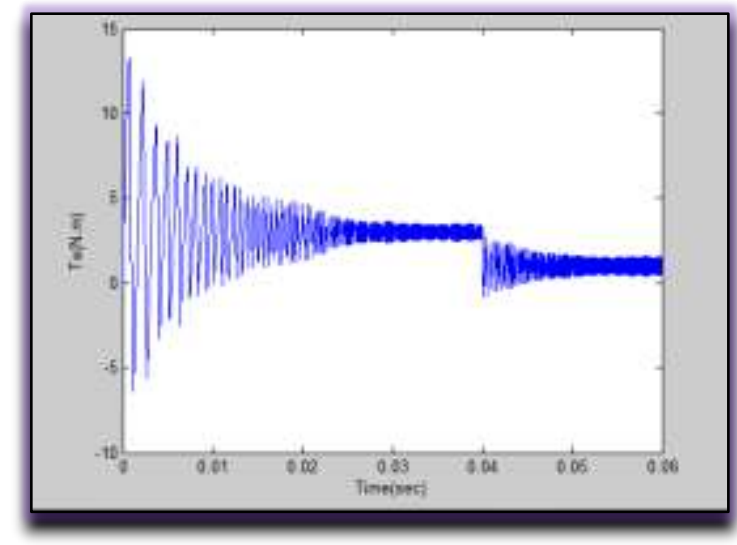

Figure 19. Electromechanical torque at mechanical faults

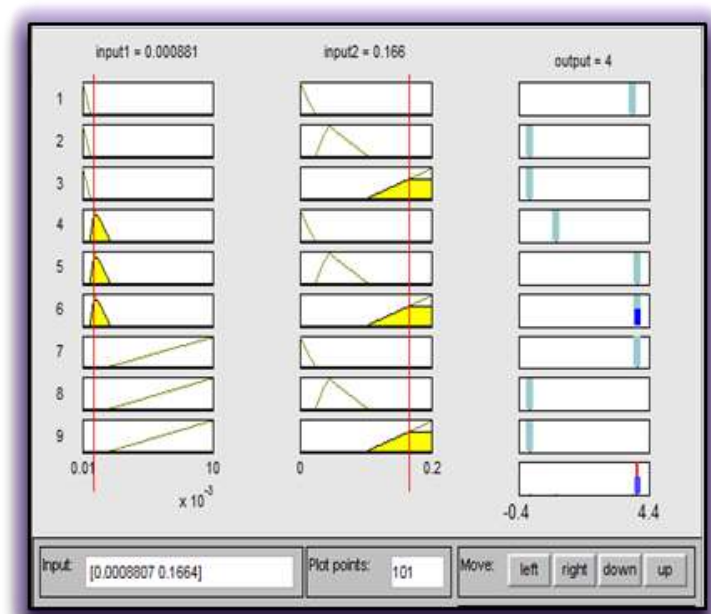

Figure 21. The results at mechanical fault (at changing Bm)

The PMSM parameters were proposed in this study can be shown in Table 1.

Table 1. PMSM Parameters

\begin{tabular}{lc}
\hline \multicolumn{1}{c}{ Constants } & Values \\
\hline Stator resistance $\left(\mathrm{R}_{\mathrm{s}}\right)$ & 2.875 \\
q-axis inductance $\left(\mathrm{L}_{\mathrm{q}}\right)$ & $8.5 \mathrm{e}-3$ \\
d-axis inductance $\left(\mathrm{L}_{\mathrm{d}}\right)$ & $8.5 \mathrm{e}-3$ \\
Rated speed $(\omega)$ & $700 \mathrm{rad} / \mathrm{sec}$ \\
Torque $\left(\mathrm{T}_{\mathrm{e}}\right)$ & 1.05 \\
Number of poles $(\mathrm{p})$ & 4 \\
Moment of inertia $(\mathrm{J})$ & $0.8 \mathrm{e}-3$ \\
Flux $(\phi)$ & 0.175 \\
Friction factor $\left(\mathrm{B}_{\mathrm{m}}\right)$ & 0 \\
\hline
\end{tabular}

The training data in first and second parts of the intelligent model can be shown in Table 2 and 3 respectively. 
Table 2. Training Data of the First Model at (Healthy, Electrical and Demgnetization Faults)

\begin{tabular}{|c|c|c|c|c|}
\hline $\begin{array}{l}\text { Stator resistance } \\
\text { (Rs) in } \Omega\end{array}$ & $\begin{array}{c}\text { Winding quadrature } \\
(\mathrm{Lq}) \text { in } \mathrm{H}\end{array}$ & Flux linkage $(\phi)$ & Condition of machine & $\mathrm{NO}$ \\
\hline $\begin{array}{c}0<\text { Rs }<2 \\
((\mathrm{RL}))\end{array}$ & $\begin{array}{c}8 \mathrm{e}-3<\mathrm{Lq}<12 \mathrm{e}-3 \\
\mathrm{LM}))((\end{array}$ & $\begin{array}{c}0.09<\phi<0.17 \\
((\mathrm{FM}))\end{array}$ & Electrical fault & 2 \\
\hline $\begin{array}{c}2<\mathrm{Rs}<3.5 \\
((\mathrm{RM}))\end{array}$ & $\begin{array}{c}8 \mathrm{e}-3<\mathrm{Lq}<12 \mathrm{e}-3 \\
\mathrm{LM}))((\end{array}$ & $\begin{array}{c}0.09<\phi<0.17 \\
\quad((\mathrm{FM}))\end{array}$ & Healthy & 1 \\
\hline $\begin{array}{c}3.5<\text { Rs }<10 \\
((\mathrm{RH}))\end{array}$ & $\begin{array}{c}8 \mathrm{e}-3<\mathrm{Lq}<12 \mathrm{e}-3 \\
\mathrm{LM}))((\end{array}$ & $\begin{array}{c}0.09<\phi<0.17 \\
\quad((\mathrm{FM}))\end{array}$ & Electrical fault & 2 \\
\hline $\begin{array}{c}2<\mathrm{Rs}<3.5 \\
((\mathrm{RM}))\end{array}$ & $\begin{array}{c}0<\mathrm{Lq}<7 \mathrm{e}-3 \\
\mathrm{LL}))((\end{array}$ & $\begin{array}{c}0.09<\phi<0.17 \\
\quad((\mathrm{FM}))\end{array}$ & Electrical fault & 2 \\
\hline $\begin{array}{c}2<\mathrm{Rs}<3.5 \\
((\mathrm{RM}))\end{array}$ & $\begin{array}{c}2 \mathrm{e}-3<\mathrm{Lq}<5 \mathrm{e}-2 \\
\quad((\mathrm{LH}))\end{array}$ & $\begin{array}{c}0<\phi<9 \mathrm{e}-2 \\
\text { FL }\end{array}$ & Electrical fault & 2 \\
\hline $\begin{array}{c}2<\text { Rs }<3.5 \\
((\mathrm{RM}))\end{array}$ & $\begin{array}{c}8 \mathrm{e}-3<\mathrm{Lq}<12 \mathrm{e}-3 \\
((\mathrm{LM}))\end{array}$ & $\begin{array}{c}0<\phi<9 \mathrm{e}-2 \\
((\mathrm{FL}))\end{array}$ & $\begin{array}{l}\text { Demagnetization } \\
\text { fault }\end{array}$ & 3 \\
\hline $\begin{array}{c}2<\mathrm{Rs}<3.5 \\
((\mathrm{RM}))\end{array}$ & $\begin{array}{c}8 \mathrm{e}-3<\mathrm{Lq}<12 \mathrm{e}-3 \\
((\mathrm{LM}))\end{array}$ & $\begin{array}{c}0.09<\phi<0.17 \\
\quad((\mathrm{FM}))\end{array}$ & $\begin{array}{l}\text { Demagnetization } \\
\text { fault }\end{array}$ & 3 \\
\hline $\begin{array}{c}2<\mathrm{Rs}<3.5 \\
((\mathrm{RM}))\end{array}$ & $\begin{array}{c}8 \mathrm{e}-3<\mathrm{Lq}<12 \mathrm{e}-3 \\
((\mathrm{LM}))\end{array}$ & $\begin{array}{c}0.09<\phi<0.17 \\
\quad((\mathrm{FM}))\end{array}$ & $\begin{array}{l}\text { Demagnetization } \\
\text { fault }\end{array}$ & 3 \\
\hline $\begin{array}{c}2<\mathrm{Rs}<3.5 \\
\quad((\mathrm{RM}))\end{array}$ & $\begin{array}{c}8 \mathrm{e}-3<\mathrm{Lq}<12 \mathrm{e}-3 \\
((\mathrm{LM}))\end{array}$ & $\begin{array}{c}0.17<\phi<0.2 \\
((\mathrm{FH}))\end{array}$ & $\begin{array}{l}\text { Demagnetization } \\
\text { fault }\end{array}$ & 3 \\
\hline
\end{tabular}

Where RL (low resistance), RM (medium resistance), RH (high resistance), LL (low q-axis inductance), LM (medium q-axis inductance), LH (high q-axis inductance), FL (low flux), FM (medium flux), FH (high flux).

Table 3. Training Data of the Second Model at (Healthy and Mechanical Fault)

\begin{tabular}{cccc}
\hline Moment of inertia $(\mathrm{J})$ & Friction factor $(\mathrm{Bm})$ & Condition of machine & No. \\
\hline $0<\mathrm{J}<5 \mathrm{e}-4$ & $0<\mathrm{Bm}<0.02$ & Mechanical fault & 4 \\
$\mathrm{JL}$ & $\mathrm{BL}$ & Healthy & 1 \\
$6 \mathrm{e}-4<\mathrm{J}<18 \mathrm{e}-4$ & $0<\mathrm{Bm}<0.02$ & $\mathrm{BL}$ & \\
$\mathrm{JM}$ & $0<\mathrm{Bm}<0.02$ & Mechanical fault & 4 \\
$2 \mathrm{e}-3<\mathrm{J}<1 \mathrm{e}-2$ & $\mathrm{BL}$ & Mechanical fault & 4 \\
$\mathrm{JH}$ & $0.02<\mathrm{J}<0.09$ & Mechanical fault & 4 \\
$6 \mathrm{e}-4<\mathrm{J}<18 \mathrm{e}-4$ & $\mathrm{BM}$ & & \\
$\mathrm{JM}$ & $0.1<\mathrm{Bm}<0.2$ & $\mathrm{BH}$ & \\
$6 \mathrm{e}-4<\mathrm{J}<18 \mathrm{e}-4$ & & & \\
$\mathrm{JM}$ & & & \\
\hline
\end{tabular}

Where JL (low inertia), JM (medium inertia), JH (high inertia), BL (low friction), BM (medium friction), BH (high friction). Figure 22 show the membership of detector for data shown in Table 2-3.

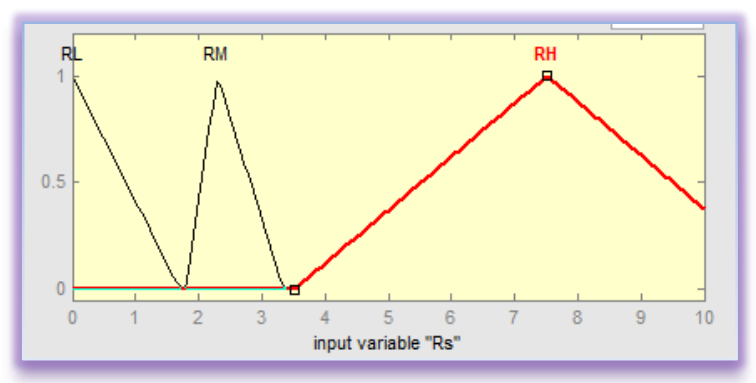

Figure 22. (a). Input Variable $\mathrm{R}_{\mathrm{s}}$ (RH High Resistance)

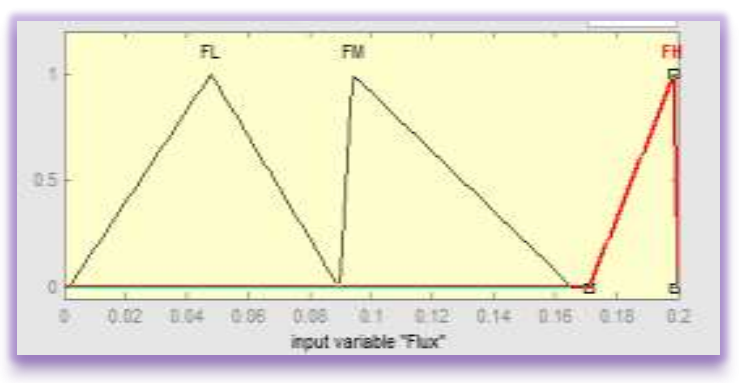

Figure 22. (b). Input Variable Flux (FH (High Flux)) 


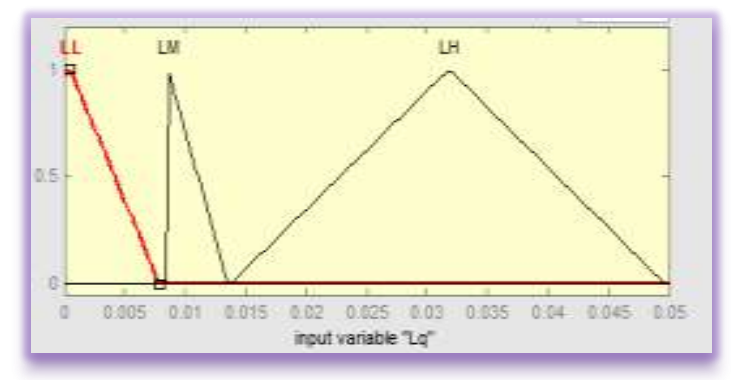

Figure 22. (c). Input Variable $\mathrm{L}_{\mathrm{q}}$

LL (low q-axis inductance)

Figure 22. Membership of Fuzzy Detector

\section{EVALUATION RESULTS AND DISCUTION}

The diagnostic process of the faults are automated using ANFIS-based agents; simulation results of ANFIS detector can be evaluated by three steps:

\subsection{Input data}

The data in Tables 4 and 5 represent the parameters of PMSM that include (RS, Lq, flux $(\phi))$ for first part of the model and $\left(\mathrm{J}, \mathrm{B}_{\mathrm{m}}\right)$ for second part. When these parameters are normal, PMSM run at healthy conditions but any change occurs in these parameters under unpredictable reasons leading to occur faults. These faults depends on the natural of changing which can be shown in Tables 4 and 5.

Table 4. Parameters of PMSM $\left(\mathrm{R}_{\mathrm{S}}, \mathrm{L}_{\mathrm{q}}, \phi\right)$ at Electrical and Demagnetization Faults

\begin{tabular}{ccccc}
\hline $\mathrm{R}_{\mathrm{s}}$ & $\mathrm{L}_{\mathrm{q}}$ & $\phi$ & Conditions of PMSM & No. \\
\hline Normal & Normal & Normal & healthy & 1 \\
low & Normal & Normal & Electric fault & 2 \\
High & Normal & Normal & Electric fault & 2 \\
Low & Low & Normal & Electric fault & 2 \\
Low & High & Normal & Electric fault & 2 \\
Normal & Low & Normal & Electric fault & 2 \\
Normal & High & Normal & Electric fault & 2 \\
High & Low & Normal & Electric fault & 2 \\
High & High & Normal & Electric fault & 2 \\
Normal & Normal & Low & Demagnetization fault & 3 \\
Normal & Normal & High & Demagnetization fault & 3 \\
\hline
\end{tabular}

Table 5. Parameters of PMSM (J, $\left.\mathrm{B}_{\mathrm{m}}\right)$ at Mechanical Fault

\begin{tabular}{cccc}
\hline Moment of inertia $(\mathrm{J})$ & Friction factor $(\mathrm{Bm})$ & Condition of machine & No. \\
\hline Normal & Normal & Healthy & 1 \\
Low & Normal & Mechanical fault & 4 \\
High & Normal & Mechanical fault & 4 \\
Normal & High (up to zero) & Mechanical fault & 4 \\
Low & High & Mechanical fault & 4 \\
high & High & Mechanical fault & 4 \\
\hline
\end{tabular}

\subsection{Evaluation of parameters by ANFIS}

ANFIS technique is a first order sugeuo type fuzzy system which consists of five layers feed forward technique that include each neural learning algorithms and fuzzy reasoning, the neural structure consists of nodes which are connect together by direct links in order to obtain one node for output. ANFIS training the three memberships functions (Mfs) for each input (parameters of PMSM) and output ((conditions of motor(healthy or faults)) by build (27 rules (if- then)) for first part of model to detect and diagnosis electric and demagnetization faults while (9 rules ) for second part to diagnosis mechanical faults, these memberships can be shown in Figure 22. 


\subsection{Conditions of PMSM}

After testing and training the data by ANFIS to obtain output of the detector (one node) refers to the conditions of PMSM (healthy condition or fault conditions) and diagnosis to the type of these faults (electrical, demagnetization and mechanical). These conditions can be shown in Table 6. The evaluation results by ANFIS can be shown in Figure 23.

Table 6. Type and Number of Fault

\begin{tabular}{cc}
\hline $\begin{array}{c}\text { Output of detector } \\
\text { (conditions of PMSM) }\end{array}$ & No. \\
\hline Healthy & 1 \\
Electrical fault & 2 \\
Demagnetization fault & 3 \\
Mechanical fault & 4 \\
\hline
\end{tabular}

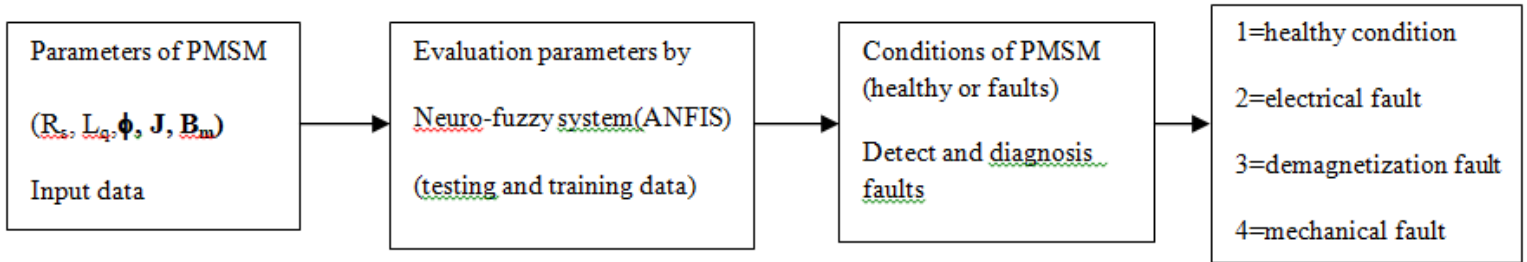

Figure 23. The evaluation results by ANFIS

\section{CONCLUSION}

In our work, we have demonstrated an intelligent technique to detect and diagnoses various types of faults that occur in PMSM (electrical, mechanical and demagnetization).This technique gives a good performance, effective and tool by performing neuro-fuzzy network. The simulation results present the successful way to detect electrical, mechanical and demagnetization faults in order to decrease maintenance cost, increase protection, and increase efficiency of the machine.

\section{REFERENCES}

[1] Yao Da, Xiadong Shi, and Mahesh Krishnamurthy, "A New Approach to Fault Diagnostics for Permanent Magnet Synchronous Machines Using Electromagnetic Signature Analysis," IEEE Transactions on Power Electronics, vol. 28, no.8, August 2013.

[2] Mustafa EKER, Mehmet AKAR, "Eccentricity Fault Diagnosis in a Permanent Magnet Synchronous Motor under Nonstationary Speed Conditions," Turkish Journal of Electrical Engineering and Computer Sciences, vol. 25, 1881-1893, 2017.

[3] Ertugryl N. Soong W. Dostal G., Saxon D.,"Faults tolerant motor drive system with redundancy for critical applications," proceedings of the IEEE Power Electronics specialists conference 2002, pp 1457-1462, 2002.

[4] Mehmet AKER, Mahmut HEKIM, and Umut ORHAN, "Mechanical Fault Detection in Permanent Magnet Synchronous Motors using Equal Width Discretization-based Probability Distribution and a Neural Network Model," Turkish Journal of Electrical Engineering and Computer Sciences, vol. 23, pp. 813-823, 2014.

[5] J.Rosero, L.Romeral, E.Rosero,J. Urresty,"Fault Detection in Dynamic Conditions by means of Discrete Wavelet Decomposition for PMSM running under Bearing Damage," IEEE Applied power Electronics Conference and Exposition, 15-19, Feb., 2009.

[6] G.B. Kliman, W.T. Premerlati, R.A. Roegl and D. Hoeweler,"A new Approach to on Line fault Detection in AC Motors," IEEE Conference on Industry Applications, vol. 1, pp. 687-693, 1996.

[7] J. Sottile and J. K. Kohler, "An on-line method to detect incipient failure of turn insulation in random-wound motors," Proceeding of IEEE Transactions on Energy Conversion, vol.8, no. 4, 1993.

[8] J. Penman, H. G. Sedding, B. A. Lloid, and W. T. Fink, "Detection and location of inter-turn short circuits in the stator windings of operating motors," Proceeding of IEEE Transactions on Energy Conversion, vol. 9, no. 4, pp. 652-658, 1994.

[9] A. Abraham and Baikunth Nath, " Hybrid Intelligent Systems: A Review of a decade of Research", School of Computing and Information Technology.

[10] Abbas H. Abbas, Alaa A. AL-Saffar, and Dhiaa K.Shary,"Bearing Fault Diagnosis in Direct Torque Control Perment Magnet Synchronous motors Based on Discrete Wavelet Transform and Artificial Neural Network," International Journal of Advances and in Engineering and Technology, vol. 7, no. 2, pp.314- 315, May 2014. 
[11] Praveen Kumar, Anurag Singh Tomer,"Modeling and Simulation of PMSM Drive with Fuzzy Logic Controller", International Journal of Modern Engineering Research (IJMER), vol. 3, no. 4, pp 2492-249, Jul-Aug. 2013.

[12] VikasKumer, Dr.SSohal,"Fuzzy Neural Networks," International Journal of Computer Science and Management Studies, vol. 12, no. 3, September, 2012.

[13] Laxminarayan Sonwani, Dr. Dharmendra Kumar Singh, Durga Sharma," Simulation of Transformer for fault Discrimination using Wavelet Transform and Neural Network," International Journal of Science, Engineering and Technology Research (IJSETR), vol. 4, no. 7, July 2015.

[14] DetlefNauck, "Neuro-Fuzzy System Review and Prospects," fifth European Congress on Intelligent Techniques and Soft Computing (EUFIT 97), Aachen, Sep,8-11, 1997, pp. 1044-1053.

[15] Mouloud Azzedine Denai and Sid Ahmed Attia, "Fuzzy and Neural Control of an Induction motor," Int. J. Appl. Math. Comput. Sci., vol. 12, no.2, 2002, pp 221-232.

[16] Mohamed A. Awadallah, Medhat M. Morcos,Suresh Gopalakrishnan and Thomas W. Nehal, "Aneuro-fuzzy Approach to Automatic Diagnosis and Location of stator Inter-Turn Fault in CSI-Fed PM Brushless DC Motors," IEEE Transaction on Energy conversion, nol. 20, no. 2, Jun 2005.

[17] K. Naga Sujatha, K. Vaisakh, "Implementation of Adaptive Neuro Fuzzy Inference System in Speed Control of Induction Motor Drives," J. Intelligent Learning Systems \& Applications, vol. 2, pp. 110-118, 2010.

[18] V.Jayalakshmi , M.V.Ramesh, "Diagnosis methods of stator short circuit faults of BLDC Motor Using Adaptive Neuro-fuzzy Inference (ANFIS) system and Wavelet," IOSR Journal of Electrical and Electronics Engineering (IOSR-JEEE), vol. 10, no. 5, pp. 21-29, Sep-Oct. 2015.

[19] PrashantW.Thmke, Nishant M. Yewale, Uttam B. Vaidya and Prashant Asutkar, "Faults Associated with Permanent Magnet Synchronous Motor," International Journal of core Engineering and Management (IJCEM), vol. 2, no. 3, June 2015.

[20] Jongman Hong, Doosoo Hyun, Tae-june Kang, Sang Bin Lee, and Anton Haumer, "Detection and Classification of Rotor Demagnetization and Eccentricity faults for PM Synchronous motors," IEEE Transaction on industry applications, vol. 48, no. 3, May/June 2012.

[21] W.Ie Roux, R. G. Harleyand T. G. Habetler " Detection Rotor Faults in low Power Permanent magnet Synchronous Machine," IEEE Transaction on power Electr. vol. 22, no. 1, pp. 322-328, June 2007.

[22] Y.Shenboo and T.Renyuan,"Electromagnetic and mechanical characteristics of noise and vibration in permanentmagnet synchronous machines," IEEE Trans. Magn., vol. 42, no. 4, pp. 1335-1338, 2006.

[23] Ali Rohan, Mohammed Rabah, and Sung H. Kim,"An Integrated Fault Detect and Identification System for Permanent Magnet Synchronous Motor in Electric vehicles," International Journal of Fuzzy Logic and Intelligent Systems, vol. 18, no. 1, pp. 20-28, March 2018.

[24] Mehmet Akar, Sezai Taskin, Sehat Seker, Ilyas Cankaya" Detection of Static Eccentricity for Permanent Magnet Synchronous motors using the Coherence analysis," Turk J Elect Eng\& Comp Sci, vol. 18, no. 6, 2010. 